\title{
Adaptive Placement of the Pseudo-boundaries improves the Conditioning of the Inverse Problem
}

\author{
Judit Chamorro-Servent ${ }^{1,2,3}$, Laura Bear ${ }^{1,4,5}$, Josselin Duchateau ${ }^{1,4,5}$, Corentin Dallet ${ }^{1,4,5}$, \\ Yves Coudière ${ }^{1,2,3}$, Rémi Dubois ${ }^{1,4,5}$ \\ ${ }^{1}$ IHU Liryc, Electrophysiology and Heart Modeling Institute, foundation Bordeaux Université, \\ Pessac-Bordeaux, France \\ ${ }^{2}$ CARMEN Research Team, INRIA, Bordeaux, France \\ ${ }^{3}$ Univ. Bordeaux, IMB, UMR 5251, CNRS, INP-Bordeaux, Talence, France \\ ${ }^{4}$ Univ. Bordeaux, CRCTB, U1045, Bordeaux, France \\ ${ }^{5}$ INSERM, CRCTB, U1045, Bordeaux, France
}

\begin{abstract}
Meshing the heart and measurement surfaces can be time consuming, especially when dealing with complicated geometries or cardiac motion. To overcome this, a meshless method based on the method of fundamental solutions (MFS) has been adapted to non-invasive electrocardiographic imaging (ECGI). In the MFS, potentials are expressed as a summation over a discrete set of virtual point sources placed outside of the domain of interest (named 'pseudo-boundary').

It is well-known that optimal placement of the pseudoboundary can improve the efficacy of the MFS. Despite this, there have been no attempts to optimize their placement in the ECGI problem as far as we are aware.

In the standard MFS, the sources are placed in two pseudo-boundaries constructed by inflating and deflating the heart and torso surfaces with respect to the geometric center of the heart. However, for some heart-torso geometries, this geometric center is a poor reference. We here show that adaptive placement of the pseudoboundaries (depending on the distance between the torso electrodes and the nearest heart locations) improves the conditioning of the inverse problem, making it less sensitive to the regularization process.
\end{abstract}

\section{Introduction}

A meshless approach that employs the method of fundamental solution (MFS) has been adapted to noninvasive electrocardiographic imaging (ECGI) to overcome some of the issues of the classical boundary element method (BEM), simplifying the forward problem but nonetheless providing accurate representations of the cardiac source [1]. In the MFS, the potentials are expressed as a linear combination of Laplace fundamental solutions over a discrete set of virtual source points placed outside of the domain of interest.

One disadvantage of the MFS is that it relies on choosing the locations of the virtual sources surrounding the domain boundary under consideration. The illposedness of the MFS problem depends on the placement of these virtual sources [2]. However, MFS optimization is a subject of ongoing research, and it has not been applied previously for the ECGI problem [1-2].

Most of the optimization approaches used for other fields: i) are developed for bi-dimensional examples, ii) require non-linear optimization, hence losing the major attractive feature of the MFS, its simplicity and easy implementation, iii) are only useful for systems with specific boundary conditions different from ours [2].

In the standard ECGI MFS method [1], the sources corresponding to the heart's surface are located on a pseudo-boundary obtained by deflating the heart by a factor 0.8. Similarly, the sources corresponding to the torso's surface are located on a pseudo-boundary obtained by inflating the torso by a factor of 1.2. Both, inflation and deflation are done relative to the geometric center of the heart. The choice of the numerical factors employed based on empirical observation in realistic human-shaped torso tank experiments, where the most accurate reconstructions were obtained for deflating by a factor 0.6-0.9 and inflating by a factor 1.1-1.5 [1].

The ECGI is innately ill-posed and small perturbations on the meshes can result in large effect on the solution. MFS employs solutions of Laplace's equations in the whole space and that inherently depend on the distance between the sources and the locations where the potentials are evaluated. This is particularly interesting for the sources corresponding to the torso, since they are far away from the heart surface. Furthermore, for structural heart diseases [3], where we can find regions of deformity, the geometric center may not be the optimal reference for 
inflating/deflating the torso and heart surfaces.

We therefore focussed on the optimization of the placement of the sources corresponding to the torso surface. We hypothesize that adaptive placement of these sources will better condition the inverse problem and improve reconstructions.

Singular Value Analysis (SVA) and discrete Picard condition (DPC) provide a means of investigating the relationship between outputs and inputs and to determine the number of independent source values that can be identified from noisy measurement data. These tools have been successfully used to optimize experimental parameters in other fields, such as fluorescence diffuse optical tomography [4]. We firstly propose a new distribution of MFS sources. Secondly, we use a simple technique based on SVA and DPC to optimize the distance where these sources must be placed, and reaffirm our hypothesis that optimal placement of the sources at the torso surface improves the stability of the solution, making the inverse problem less sensitive to regularization. Finally, we compared the effect of the new sources with a dataset from a patient with a myocardial scar presenting.

\section{Methods}

\subsection{Standard MFS method}

In the MFS, an approximate solution is represented in the form of linear superposition of source functions (Laplace fundamental solutions) located on a pseudoboundary (set of virtual sources) over an auxiliary surface.

By using Dirichlet and Neumann conditions at the torso boundary as defined in [1] we obtain a linear system:

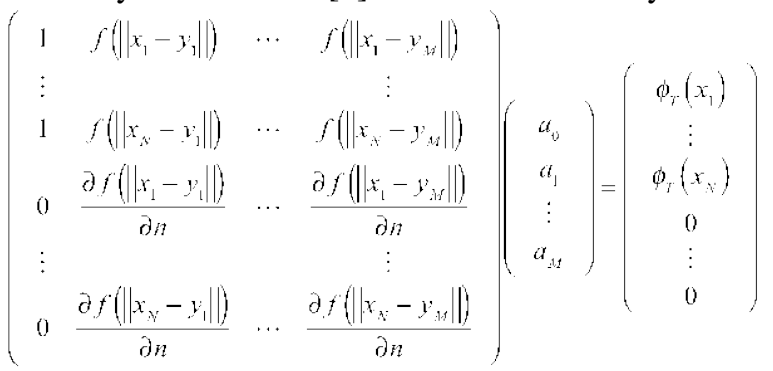

(1)

where $x_{i}(i=1 . . N)$ are the location of $N$ electrodes on the torso, $y_{j}(j=1 . . M)$ the location of the virtual sources on the torso and heart pseudo-boundaries (so $M=M_{T}+M_{H}$ ), $f\left(\left\|x_{i}-y_{j}\right\|\right)=1 /\left(4 \pi\left\|x_{i}-y_{j}\right\|\right)$ the fundamental solution of Laplace's equation in 3D, $a_{0}$ the constant component of the torso potentials $\left(\phi_{T}(x)\right)$, and $a_{j}$ the coefficient of a virtual source at location $y_{j}$ over an auxiliary surface $\hat{\Gamma}$. Being $\hat{\Gamma}=\hat{\Gamma}_{T} \cup \hat{\Gamma}_{H}$, the union of both auxiliary surfaces.

The virtual source coefficients of (1) are computed by using zero-order Tikhonov regularization and the potential $\phi(x)$ at any location $x$ of the domain can be expressed as:

$\phi(x)=a_{0}+\sum_{j=1}^{M} a_{j} f\left(\left\|x-y_{j}\right\|\right), x \in \Omega, y_{j} \in \hat{\Gamma}$

\subsection{Optimization of the distribution of the torso pseudo-boundary}

The Laplace fundamental solution strongly depends on the Euclidian distance $\left(\left\|x_{i}-y_{i}\right\|\right)$ between sources $y_{i}$ and locations $x_{i}$ where the potentials are evaluated, $i=1, \cdots, M_{T}$.

We hypothesize that a placement of the torso pseudoboundary, inflating the torso with respect to the closest points of the heart surface instead of the geometric isobarycenter, will better condition our problem. The following method is used to test this: i) for each electrode $x_{i}$ on the torso, we find the location of closest point on the heart surface, i.e., the $h_{d_{\min }}$ such that $d_{\min }=\min \left(\left\|x_{i}-h_{k}\right\|_{2}\right)$, $k=1, \cdots, M_{H}$, ii) We construct the location of the virtual source $y_{i}$ related to the torso electrode $x_{i}$ as $y_{i}=x_{i}+R_{T}\left(x_{i}-h_{d_{\min }}\right)$, iii) We use SVA to find the ratio $R_{T}$ that makes our problem better conditioned, i.e. that improves the SV decay.

\subsection{SVA and DPC as tools to optimize the placement of sources}

By doing a singular value decomposition (SVD) of the matrix of (1), the projection of the data on the left singular vectors $U^{T}$ is related to the projection of the sources on the right singular vectors $V^{T}$ by means of the singular values (diagonal values of the matrix $S$ ):

$$
U^{T}\left(\begin{array}{c}
\phi_{T} \\
0 \\
\vdots \\
0
\end{array}\right)=S V^{T}\left(\begin{array}{c}
a_{0} \\
a_{1} \\
\vdots \\
a_{M}
\end{array}\right)
$$

Rather than the condition number of the matrix of a linear system, the rate of decrease of the singular values (SVs) is a better indication of the conditioning of the problem [5]. Furthermore, the discrete Picard's condition (DPC) provides an objective assessment of the illposedness of the entire problem relating the matrix information (by means of the SVs) with the measurements.

The Tikhonov zero order solution (for a regularization parameter $\alpha$ ) can be expressed in terms of the SVD as:

$$
a_{\alpha}=\sum_{i=1}^{\min (M, N)} \frac{\sigma_{i}^{2}}{\sigma_{i}^{2}+\alpha^{2}} \frac{u_{i}^{T} b}{\sigma_{i}^{2}} v_{i}
$$

The DPC provides an idea of the ratio between the decay of the singular values $\left(\sigma_{i}\right)$ of the MFS matrix and the decay of the coefficients of the right-hand side $(b)$, when expressed in terms of the left singular vectors of the 
matrix $\left(\left|\boldsymbol{u}_{i}^{T} \boldsymbol{b}\right|\right)$. An optimal regularization parameter for Tikhonov solution must fulfill the DPC [5]; that is, the $\sigma_{\boldsymbol{i}}$ above the regularization parameter (useful SVs) must decay to zero slower than the corresponding $\left|\boldsymbol{u}_{i}^{\boldsymbol{T}} \boldsymbol{b}\right|$. Therefore, the percentage of useful SVs can be used as a measure of posedness of an inverse problem.

\section{Results}

\subsection{Singular value analysis}

We studied the decay rate of the SVs of the MFS matrices regarding the standard and new distributions of virtual sources for different $R_{T} \in[0.1, \ldots, 0.5]$. First, we compared them for two different patients: a patient having LBBB (a-b) and a patient presenting a scar (c-d). Afterwards, we placed the heart geometries of (a) and (c) inside an artificial torso geometry by using the same center point in both cases and we repeat the study, with the purpose of studying the effect of the new sources depending on the heart geometry.

For all torso-heart geometries, the first SVs of the MFS using the new distribution of sources decay slower than for the standard MFS. Besides, when using the same torso geometry (e and g), the new distribution has more impact for the case involving the heart with scar presenting a ventricular aneurysm (g-h).

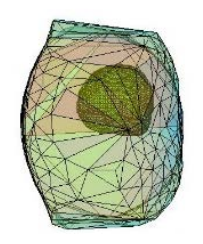

(a)

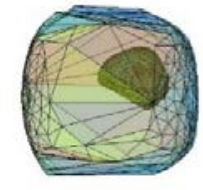

(c)

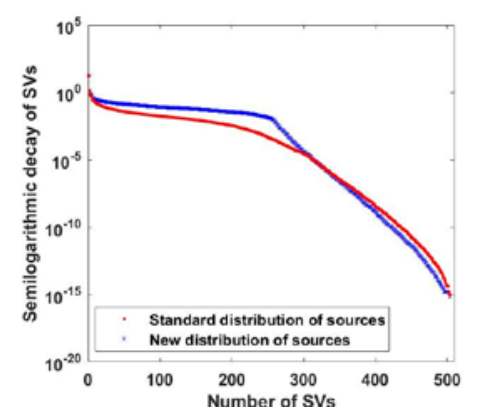

(b)

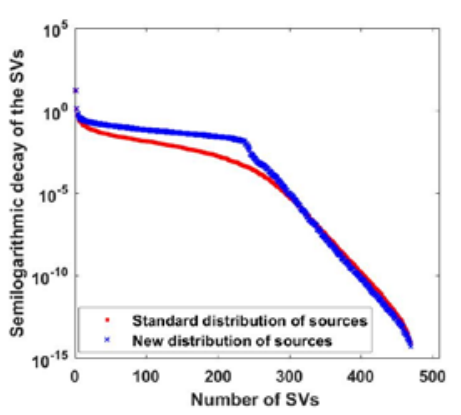

(d)

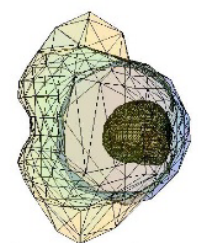

(e)

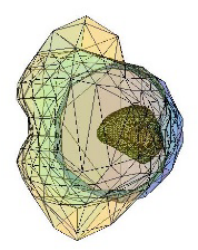

(g)

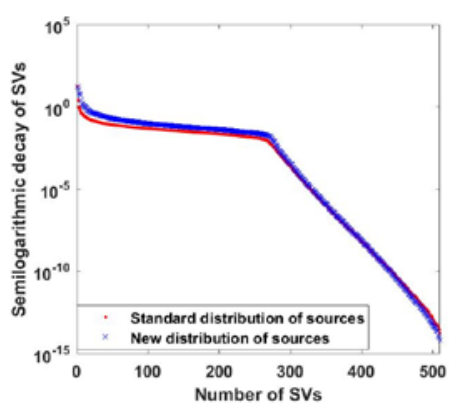

(f)

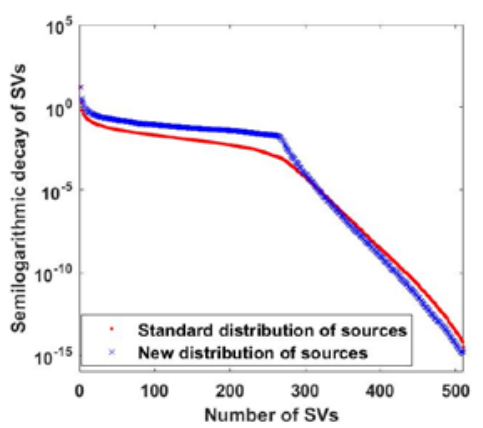

(h)
Fig. 1: Left - Upper view of the different geometries used, right - related decay rate of SVs of the matrices corresponding to the standard and new distribution of sources for the best $R_{T}$ (i.e. the $R_{T}$ providing better conditioning of the MFS matrix). Geometries: (a) torsoheart of patient with LBBB, (c) torso-heart of patient with a scar, (e) heart geometry of (a) in an artificial torso, (g) heart geometry of (c) in the same artificial torso than (e).

\subsection{Discrete Picard condition}

For the cases (a-b) and (c-d) having the measured potentials at torso, we studied the DPC for the standard and new distribution of sources. To do that, we plotted the semi-logarithmic SVs $\left(\sigma_{i}\right)$ decay on blue dot points, the respective $\left|\boldsymbol{u}_{i}^{T} \boldsymbol{b}\right|$ in red cross and the quotient between both in yellow circles.
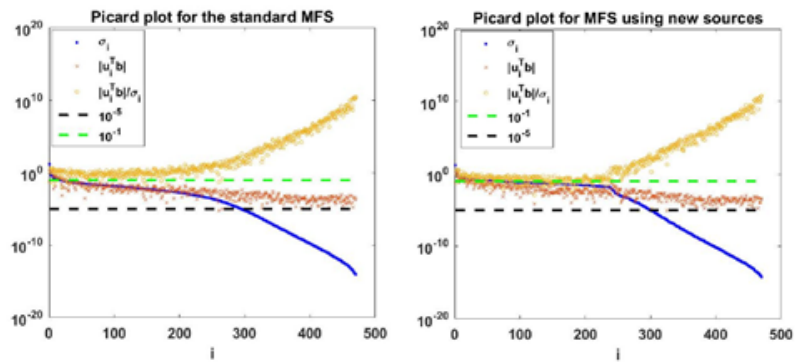

Fig. 2: DPC plot example for the scar patient (case cd of fig. 1), for an instant of time $t_{k}$ of interest. Left: for the standard MFS problem. Right: for the MFS problem 
resulting from the new distribution of sources.

For the particular case of a patient referred having a scar (Fig.1.c), we can see that SVs start to decay faster to zero than their respective $\left|u_{i}^{T} b\right|$ : for $\mathrm{i}>180(38.30 \%$ useful SVs) in the standard MFS and for i>235 (50\% useful SVs) in the MFS with new distribution of sources.

The DPC and the decay of the first SVs ( $i \leq 250$ in all cases) seems to indicate that the new sources made our problem less sensitive to the regularization parameter chosen. To prove it we reconstructed the signals at the heart for different regularization parameters of zeroorder Tikhonov, $\alpha \in\left[10^{-5}, 10^{-1}\right]$.
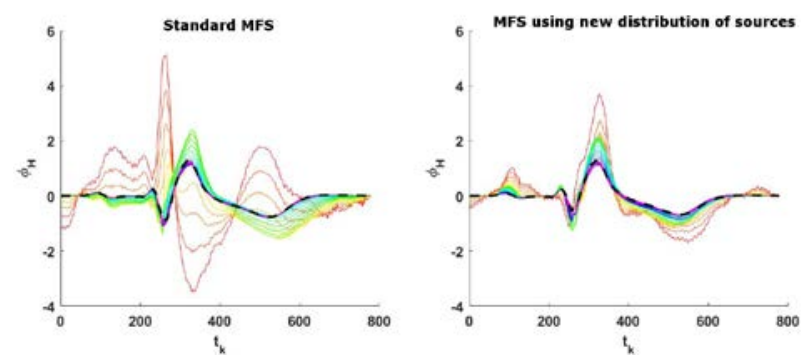

Fig. 3: Temporal heart reconstructed potentials for one point of the heart. Left: By using old distribution of sources. Right: By using new distribution. The different colors show the different regularization parameters used. The dash black line highlight the solution chosen in practice.

Finally, we computed the $\min (d V / d T)$ for the different solutions provided by using the regularization parameters fulfilling the DPC. We found a variability of $22.0038 \mathrm{~ms}$ for the standard distribution, against $16.1386 \mathrm{~ms}$ for those using the new sources.

\section{Discussion and conclusions}

While the efficacy of the MFS solution has shown dependence on the placement of its pseudo-boundary [2], there has been no attempts at optimizing its placement in the ECGI problem [1-2]. SVA and DPC bring the interaction of the dependence between the placement of the virtual sources and the posedness of the MFS problem into clearer focus. Both techniques share with MFS its major attractive feature: its simplicity and easy implementation, and they can be successfully used to improve the distance and source distributions.

This work has the aim to test the hyphothesis that an optimal placement of the torso virtual sources (by doing the projection of the closest heart locations for each electrode), improves the posedness of the inverse problem compared to the standard approach. The percentage of useful SVs provided by DPC is an indication of the conditioning of a problem. For the patient with scar we obtained $50 \%$ useful SVs by using the new distribution of sources against $38.30 \%$ using the standard distribution. Furthermore, the reconstructed potentials at the heart and the variability of the activation times provided showed less dependence to the regularization parameter for the new sources than for the standard ones.

Figure 1.g-h and 1e-f, suggest that the improvement provided for the new distribution of sources is more significant for structural disease. This is likely explained by the isobarycenter used by the standard MFS to inflate and deflate the pseudo-boundaries which is a poor reference in these cases.

In this work we have only focused on the sources regarding the torso surface (further away from heart) but we hypothesize that an optimal placement of the heart virtual sources could also have some impact on the solution. Further research in this direction will be done.

\section{Acknowledgements}

This study received financial support from the French Government as part of the "Investments of the Future" program managed by the National Research Agency (ANR), Grant reference ANR-10-IAHU-04 and from the Conseil Régional Aquitaine as part of the project "Assimilation de données en cancérologie et cardiologie".

\section{References}

[1] Wang Y, Rudy Y. Application of the method of fundamental solutions to potential-based inverse electrocardiography. Annals of biomedical engineering 2006; 34(8): 1272-1288.

[2] Karageorghis A, Lesnic D, Marin L. (2011). A survey of applications of the MFS to inverse problems. Inverse Problems in Science and Engineering 2013; 19(3), 309-336.

[3] Porras A R, et al. Cardiac Deformation from ElectroAnatomical Mapping Data: Application to Scar Characterization. In: FIMH. NY, USA, 2011: 47-54.

[4] Chamorro-Servent J, et al. FDOT reconstruction and setting optimization using SVA with automatic thresholding. In: NSS/MIC. Orlando, Florida: IEEE, 2009: 2827-2829.

[5] Hansen P C. Discrete inverse problems: insight and algorithms. Philadelphia: SIAM, 2010.

Address for correspondence:

Judit Chamorro Servent

IHU Liryc, Electrophysiology and Heart Modeling Institute, foundation Bordeaux Université, F-33600 Pessac-Bordeaux, France Judit.Chamorro-Servent@ihu-liryc.fr 